\title{
AN ANALYSIS OF A FRACTAL MICHAELIS-MENTEN CURVE
}

\author{
JACK HEIDEL ${ }^{1}$ and JOHN MALONEY ${ }^{1}$
}

(Received 4 October 1995; revised 6 July 1998)

\begin{abstract}
Simple chemical reactions can be described by the Michaelis-Menten response curve relating the velocity $V$ of the reaction and the concentration $[S]$ of the substrate $S$. To handle more complicated reactions without introducing general polynomial response curves, the rate constants can be considered to be scale dependent. This leads to a new response curve with characteristic sigmoidal shape. But not all sigmoidal curves can be accurately fit with three parameters. In order to get an accurate fit, the lower part of the $\int$ shaped curve cannot be too shallow and the upper part can't be too steep. This paper determines an exact mathematical expression for the steepness and shallowness allowed.
\end{abstract}

\section{Introduction}

The simple chemical reaction

$$
E+S \stackrel{k_{1}}{\underset{k_{-1}}{\longrightarrow}} C \stackrel{k_{2}}{\longrightarrow} E+P
$$

can be described by the Michaelis-Menten equation

$$
V=\frac{V_{\max }[S]}{K_{M}+[S]}
$$

where $[S]$ is the concentration of the substrate $S$ and $V=\left|\frac{d[S]}{d t}\right|$ is the reaction velocity [6]. The response curve (2) has the characteristic concave form of Figure 1.

It is known that the Michaelis-Menten equation (2) and its fractal version (6) described in the next section are both parameter identifiable in the sense that perfect data for which there is a good fit to one of the curves uniquely determines the parameters. However it turns out that not any sigmoidal shaped curve can be fit by a fractal Michaelis-Menten curve. The steepness and shallowness of both the upper and lower parts of the curve determine whether or not this can be done.

\footnotetext{
${ }^{1}$ Department of Mathematics, University of Nebraska at Omaha, Omaha, NE 68182

(C) Australian Mathematical Society 2000, Serial-fee code 0334-2700/00
} 


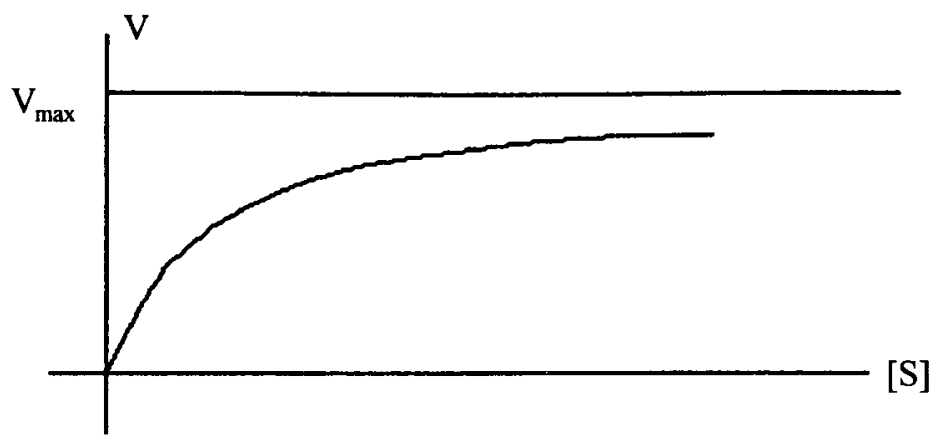

FIGURE 1.

This paper does not deal with the parameter estimation problem which simply determines the best parameter fit to any sigmoidal curve regardless of how good it is.

To introduce the methods of this paper the Michaelis-Menten equation (2) will be considered. Suppose that the two data points $\left([S]_{1}, V_{1}\right)$ and $\left([S]_{2}, V_{2}\right)$ are given.

Thus

$$
V_{1}=\frac{V_{\max }[S]_{1}}{K_{M}+[S]_{1}}, \quad V_{2}=\frac{V_{\max }[S]_{2}}{K_{M}+[S]_{2}}
$$

Solving each equation for $V_{\max }$ we obtain

$$
\frac{V_{1}\left(K_{M}+[S]_{1}\right)}{[S]_{1}}=V_{\max }=\frac{V_{2}\left(K_{M}+[S]_{2}\right)}{[S]_{2}} .
$$

Now solving for $K_{M}$ we find that

$$
K_{M}=\frac{\left(V_{2}-V_{1}\right)[S]_{1}[S]_{2}}{V_{1}[S]_{2}-V_{2}[S]_{1}}
$$

provided that the denominator is not zero, that is,

$$
V_{1} /[S]_{1} \neq V_{2} /[S]_{2} \text {. }
$$

Since $V_{1} /[S]_{1}$ and $V_{2} /[S]_{2}$ are the slopes of the lines from the origin to the data points, it is clear from the graph of (2) that (3) holds (Figure 2).

It has thus been shown that the two points on the concave curve (2) determine the two parameters uniquely. This is the best that can be accomplished. Clearly, three or more data points on (2) provide an overdetermined system and in general no exact solution will exist.

To be a little more specific, for future reference, the condition (3) should be replaced by

$$
\frac{V_{1}}{[S]_{1}}>\frac{V_{2}}{[S]_{2}}
$$




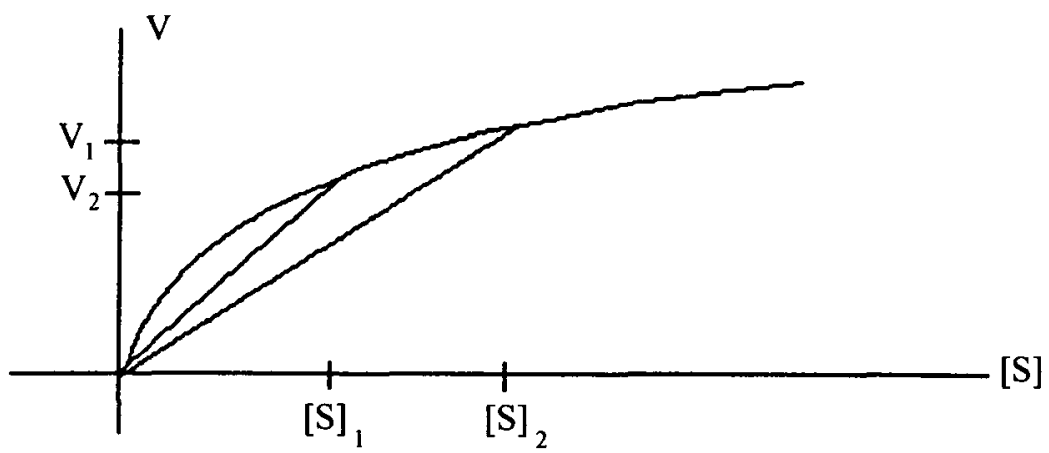

FIGURE 2.

to take into account the shape of the curve (2). In other words, given two points $\left([S]_{1}, V_{1}\right)$ and $\left([S]_{2}, V_{2}\right)$ satisfying (4), there are two numbers $V_{\max }$ and $K_{M}$ uniquely determined such that the curve (2) passes through the two given points. The analogue of condition (4) for the fractal Michaelis-Menten equation turns out to be significant for the analysis of this equation.

\section{The fractal Michaelis-Menten equation}

The simple reaction scheme leading to the Michaelis-Menten equation may be an oversimplification of the true chemical behavior. Taking into account intermediate reactions leads to a more general response equation

$$
V=\frac{\alpha_{1}[S]+\alpha_{2}[S]^{2}+\cdots+\alpha_{n}[S]^{n}}{\beta_{0}+\beta_{1}[S]+\beta_{2}[S]^{2}+\cdots+\beta_{m}[S]^{m}},
$$

considered by Wong [7]. To avoid such use of numerous parameters with only a vague and misleading chemical interpretation, Lopez-Quintela and Casado have introduced a fractal approach [4]. The idea is to consider the constants $k_{i}$ in (2) as being scale dependent,

$$
k_{i}=k_{i}^{\mathrm{eff}}=A_{i}[S]^{1-D},
$$

where $D \leq 1$ is the fractal dimension of the scaling variable $[S]$. Inserting this form into (2) yields the new response curve,

$$
V=\frac{V_{\max }^{\mathrm{eff}}[S]^{2-D}}{K_{M}^{\mathrm{eff}}+[S]}
$$


where $V_{\max }^{\text {eff }}$ and $K_{\max }^{\text {eff }}$ are new constants. Clearly (5) is a more general response curve than (2) and reduces to (2) if $D=1$. In fact, as discussed in [4], one reason for using (5) is to determine to what extent a chemical reaction is adequately described by a Michaelis-Menten response curve.

It is not hard to show that the graph of (5) has the sigmoidal form (for $0<D<1$ ) (Figure 3 ) with a single inflection point.

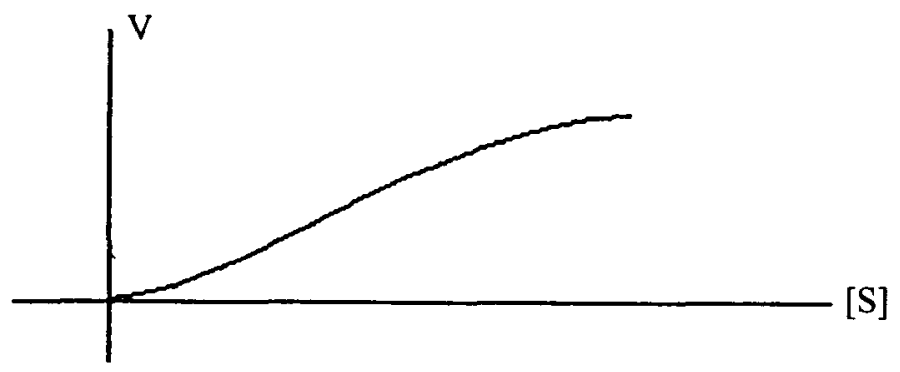

FigURE 3.

Note that the response curve (5) has three parameters, $V_{\max }^{\text {eff }}, K_{M}^{\text {eff }}$ and $D$ as opposed to the two parameters in the classical Michaelis-Menten equation. The purpose of this paper is to discuss the data fitting problem for (5). It turns out that this three parameter problem is much more complicated than the corresponding two parameter problem for (2).

Before beginning this analysis, another three parameter chemical response curve should be mentioned, namely the Hill equation

$$
V=\frac{V_{\max }[S]^{n}}{K_{M}+[S]^{n}}
$$

arising in cooperative chemical kinetics [6]. The parameter analysis problem for (6) can be discussed in a similar way, although it is interesting that the final result is different [3].

\section{The parameter analysis}

Since there are three parameters in (5), three data points are needed. Changing notation for simplicity in the lengthy calculations to follow, suppose $\left(x_{i}, y_{i}\right)$ satisfy

$$
y_{i}=\frac{M x_{i}^{n}}{Q+x_{i}}
$$

for $i=1,2,3$ where $1<n<2,0<x_{1}<x_{2}<x_{3}$ and $0<y_{1}<y_{2}<y_{3}$. The following theorem will be established. 
THEOREM 1. Let

$$
a=\frac{\ln x_{3}-\ln x_{1}}{\ln x_{3}-\ln x_{2}}>1 .
$$

Then the equations obtained by putting $\left(x_{i}, y_{i}\right)$ in (7) have a unique solution for $M$, $Q$ and $n$ if $y_{2}^{a} \leq y_{1} y_{3}^{a-1}$.

Later it will be shown that in the non-unique case there are either two solutions for $M, Q$ and $n$ or no solutions.

The proof is long and computational. Each of the data equations is solved for $n$,

$$
n=\frac{1}{\ln x_{i}} \ln \frac{y_{i}\left(Q+x_{i}\right)}{M}, \quad i=1,2,3,
$$

and, solving for $\ln M$,

$$
\ln M=\frac{\ln \left(y_{i}\left(Q+x_{i}\right)\right) \ln x_{1}-\ln \left(y_{1}\left(Q+x_{1}\right)\right) \ln x_{i}}{\ln x_{1}-\ln x_{i}}, \quad i=2,3 .
$$

Setting these two expressions for $\ln M$ equal to each other and rearranging gives

$$
\begin{aligned}
& \ln \left(Q+x_{1}\right)\left[\ln x_{3}-\ln x_{2}\right] \ln x_{1} \\
& \quad=\ln \left(Q+x_{3}\right)\left[\ln x_{1}-\ln x_{2}\right] \ln x_{1}-\ln \left(Q+x_{2}\right)\left[\ln x_{1}-\ln x_{3}\right] \ln x_{1}+k_{1},
\end{aligned}
$$

where

$$
k_{1}=\ln x_{1}\left[\ln y_{2}\left(\ln x_{3}-\ln x_{1}\right)-\ln y_{3}\left(\ln x_{2}-\ln x_{1}\right)-\ln y_{1}\left(\ln x_{3}-\ln x_{2}\right)\right] .
$$

Thus

$$
\ln \left(Q+x_{1}\right)=a \ln \left(Q+x_{2}\right)-(a-1) \ln \left(Q+x_{3}\right)+k_{2},
$$

where

$$
k_{2}=\frac{k_{1}}{\ln x_{1}\left(\ln x_{3}-\ln x_{2}\right)} .
$$

Thus

$$
\ln \left(Q+x_{1}\right)=\ln \frac{\left(Q+x_{2}\right)^{a}}{\left(Q+x_{3}\right)^{a-1}}+k_{3}
$$

where $k_{3}=\ln \frac{y_{2}^{a}}{y_{1} y_{3}^{a-1}}$.

Finally

$$
y_{1}\left(Q+x_{1}\right)=\frac{y_{2}^{a}\left(Q+x_{2}\right)^{a}}{y_{3}^{a-1}\left(Q+x_{3}\right)^{a-1}}
$$


and the original three equations (7) in $M, Q$ and $n$ have been reduced to a single equation in $Q$.

Defining

$$
f(Q)=y_{1}\left(Q+x_{1}\right) \text { and } h(Q)=\frac{y_{2}^{a}\left(Q+x_{2}\right)^{a}}{y_{3}^{a-1}\left(Q+x_{3}\right)^{a-1}},
$$

it follows that (8) has a solution whenever the curves $f(Q)$ and $h(Q)$ intersect. The character of $f$ and $h$ will now be investigated. It will first be shown that $h(Q)$ is monotone increasing and concave up. For simplicity consider

$$
g(Q)=\frac{\left(Q+x_{2}\right)^{a}}{\left(Q+x_{3}\right)^{a-1}}
$$

It is easily shown that

$$
g^{\prime}(Q)=\frac{\left(Q+x_{2}\right)^{a}}{\left(Q+x_{3}\right)^{a-1}}\left[\frac{\left(Q+x_{2}\right)+a\left(x_{3}-x_{2}\right)}{\left(Q+x_{2}\right)\left(Q+x_{3}\right)}\right]>0,
$$

for $Q+x_{1}>0$. Differentiating again, it turns out that

$$
g^{\prime \prime}(Q)=\frac{a(a-1)\left(Q+x_{2}\right)^{a-2}\left(x_{3}-x_{2}\right)^{2}}{\left(Q+x_{3}\right)^{a+1}}>0
$$

also showing that $g$, and hence $h$, is increasing and concave up, for $Q+x_{1}>0$.

Several cases must now be considered. If $y_{1}>\frac{y_{2}^{a}}{y_{3}^{a-1}}$ then $\lim _{Q \rightarrow \infty} \frac{f(Q)}{h(Q)}>1$, that is, for sufficiently large $Q, f(Q)>h(Q)$. The concavity of $h$ then requires a unique point $Q$ of intersection for $f$ and $h$ as indicated in Figure 4.

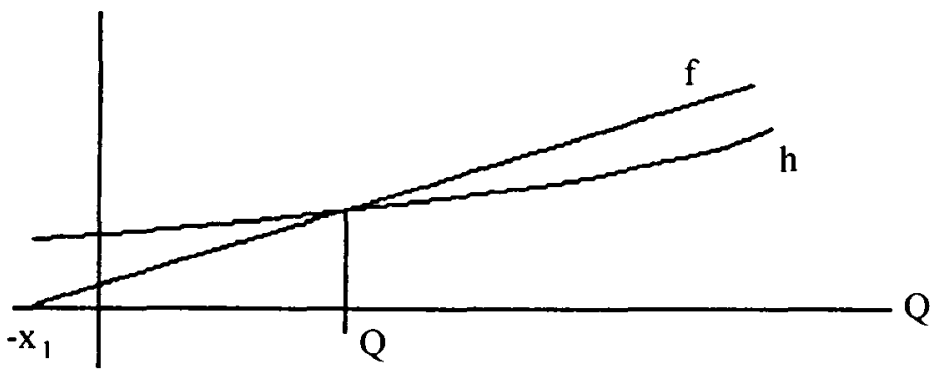

FIGURE 4 .

Next suppose that $y_{1}=\frac{y_{2}^{a}}{y_{3}^{a-1}}$. To proceed further, the asymptote as $Q \rightarrow \infty$ for the curve $h(Q)$ is needed. The equation of the asymptote is found by finding the Taylor series expansion of

$$
\frac{\left(Q+x_{2}\right)^{a}}{\left(Q+x_{3}\right)^{a-1}}=\frac{\left[R+\left(x_{2}-x_{3}\right)\right]^{a}}{R^{a-1}}, \quad \text { with } R=Q+x_{3} \text {, as } R \rightarrow \infty
$$


Let $z=1 / R$ and

$$
G(z)=\left[\frac{1}{z}+\left(x_{2}-x_{3}\right)\right]^{a}=\left[\frac{1+\left(x_{2}-x_{3}\right) z}{z}\right]^{a}, \quad \text { as } z \rightarrow \infty .
$$

Then $H(z)=\left[1+\left(x_{2}-x_{3}\right) z\right]^{a}$ satisfies $H(0)=1$ and $H^{\prime}(0)=a\left(x_{2}-x_{3}\right)$. Thus

$$
\begin{aligned}
& H(z)=1+a\left(x_{2}-x_{3}\right) z+\cdots, \quad \text { as } z \rightarrow 0, \\
& G(z)=\frac{H(z)}{z^{a}}=\frac{1}{z^{a}}+a\left(x_{2}-x_{3}\right) z^{1-a}+\cdots, \quad \text { as } z \rightarrow 0, \\
& \frac{\left[R+\left(x_{2}-x_{3}\right)\right]^{a}}{R^{a-1}}=R+a\left(x_{2}-x_{3}\right)+\cdots, \quad \text { as } R \rightarrow \infty, \\
& \frac{\left(Q+x_{2}\right)^{a}}{\left(Q+x_{3}\right)^{a-1}}=Q+x_{3}+a\left(x_{2}-x_{3}\right)+\cdots, \quad \text { as } Q \rightarrow \infty,
\end{aligned}
$$

and finally

$$
\frac{y_{2}^{a}\left(Q+x_{2}\right)^{a}}{y_{3}^{a-1}\left(Q+x_{3}\right)^{a-1}}=\frac{y_{2}^{a}}{y_{3}^{a-1}}\left[Q+x_{3}+a\left(x_{2}-x_{3}\right)\right]+\cdots, \quad \text { as } Q \rightarrow \infty,
$$

and the first term on the right-hand side is the asymptote. The asymptote's $Q$ intercept is the point

$$
Q=a\left(x_{3}-x_{2}\right)-x_{3} .
$$

It will now be shown that $a>\frac{x_{3}-x_{1}}{x_{3}-x_{2}}$ which implies that $a\left(x_{3}-x_{2}\right)-x_{3}>-x_{1}$. This is accomplished by examining the equation $a=\frac{x_{3}-x_{1}}{x_{3}-x_{2}}$ which can be rewritten as

$$
x_{3}-\left(\frac{\ln x_{3}-\ln x_{2}}{\ln x_{3}-\ln x_{1}}\right)\left(x_{3}-x_{1}\right)=x_{2} .
$$

Now consider the function

$$
\alpha(x)=x_{3}-\left(\frac{\ln x_{3}-\ln x}{\ln x_{3}-\ln x_{1}}\right)\left(x_{3}-x_{1}\right) .
$$

It turns out that $\alpha\left(x_{1}\right)=x_{1}, \alpha\left(x_{3}\right)=x_{3}, \alpha^{\prime}(x)>0$ and $\alpha^{\prime \prime}(x)<0$. This implies that $x_{2}<\alpha\left(x_{2}\right)$ for $x_{1}<x_{2}<x_{3}$ and thus

$$
x_{2}<x_{3}-\left(\frac{\ln x_{3}-\ln x_{2}}{\ln x_{3}-\ln x_{1}}\right)\left(x_{3}-x_{1}\right),
$$

which proves the assertion.

But $a\left(x_{3}-x_{2}\right)-x_{3}>-x_{1}$ implies the following alignment between the graphs of $f$ and $h$ (Figure 5).

Thus the case $y_{1}=\frac{y_{2}^{a}}{y_{3}^{a-1}}$ means that the asymptote for $h$ has the same slope as $f$ and lies strictly below $f$. Hence $h$ and $f$ have a unique point of intersection and the theorem is proved. 


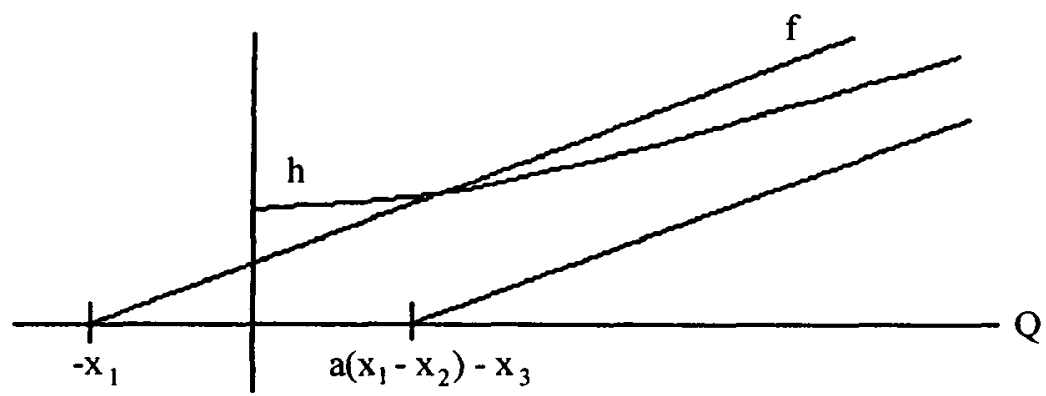

FIGURE 5.

REMARK 1. $Q$ must be positive to have a useful implementation in the fractal Michaelis-Menton equation. From the above analysis $Q>0$ exactly when $h(0)>$ $f(0)$, or for

$$
\frac{y_{2}^{a} x_{2}^{a}}{y_{3}^{a-1} x_{3}^{a-1}}>y_{1} x_{1}
$$

But $a=\frac{\ln x_{3}-\ln x_{1}}{\ln x_{3}-\ln x_{2}}$ can be rewritten as

$$
\frac{x_{3}}{x_{1}}=\left(\frac{x_{3}}{x_{2}}\right)^{a}
$$

Then (9) reduces to $\frac{y_{2}^{a}}{y_{3}^{a+1}}>y_{1}$. Hence $Q<0$ in the uniqueness region of Theorem 1 except on the boundary $y_{1}=\frac{y_{2}^{a}}{y_{2}^{a-1}}$ where $Q=0$. By similar reasoning $Q$ is always positive under the hypothesis of Theorem 2.

THEOREM 2. Suppose $y_{1} y_{3}^{a-1}<y_{2}^{a}$. If, in addition,

$$
y_{1}^{1 / a} y_{3}^{(a-1) / a} \frac{(a-1)^{(a-1) / a}}{a} \frac{x_{3}-x_{1}}{\left(x_{3}-x_{1}\right)^{(a-1) / a}\left(x_{3}-x_{2}\right)^{1 / a}}>y_{2},
$$

there are two solutions for $M, Q$ and $n$. If the inequality in (10) is reversed, there are no solutions for $M, Q$ and $n$, and if the inequality is replaced by an equality, then there is exactly one solution.

To prove the theorem, again consider

$$
y_{1}<\frac{y_{2}^{a}}{y_{3}^{a-1}},
$$




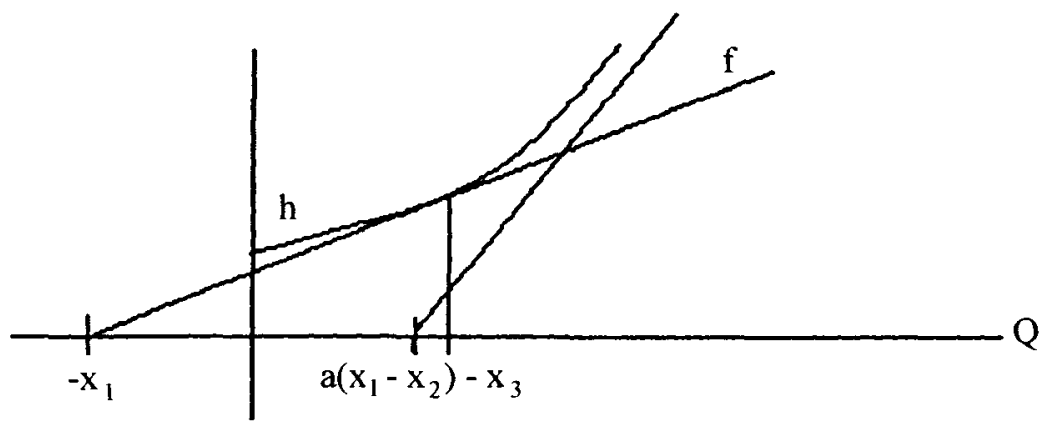

FIGURE 6.

in which case the asymptote for $h$ has a larger slope than for $f$. To resolve this case suppose that the graphs of $f$ and $g$ have a point of tangency $Q$ (Figure 6).

It is clear from the above diagram that there may be either two, one, or no solutions in the case (11). A unique solution corresponds to a point of tangency and is the dividing line between the other two possibilities.

Thus suppose that $f(Q)=h(Q)$ and $f^{\prime}(Q)=h^{\prime}(Q)$ for some point $Q$. Then

$$
y_{1}\left(Q+x_{1}\right)=\frac{y_{2}^{a}\left(Q+x_{2}\right)^{a}}{y_{3}^{a-1}\left(Q+x_{3}\right)^{a-1}}
$$

and

$$
y_{1}=f^{\prime}(Q)=h^{\prime}(Q)=\frac{y_{2}^{a}}{y_{3}^{a-1}} \frac{\left(Q+x_{2}\right)^{a}}{\left(Q+x_{3}\right)^{a-1}}\left[\frac{\left(Q+x_{2}\right)+a\left(x_{3}-x_{2}\right)}{\left(Q+x_{2}\right)\left(Q+x_{3}\right)}\right] .
$$

It follows that

$$
y_{1}\left(Q+x_{1}\right)=\frac{y_{2}^{a}}{y_{3}^{a-1}} \frac{\left(Q+x_{2}\right)^{a}}{\left(Q+x_{3}\right)^{a-1}}\left[\frac{\left(Q+x_{2}\right)+a\left(x_{3}-x_{2}\right)}{\left(Q+x_{2}\right)\left(Q+x_{3}\right)}\right]\left(Q+x_{1}\right)
$$

and finally

$$
\left[\frac{\left(Q+x_{2}\right)+a\left(x_{3}-x_{2}\right)}{\left(Q+x_{2}\right)\left(Q+x_{3}\right)}\right]\left(Q+x_{1}\right)=1 .
$$

Expanding and solving for $Q$ yields

$$
Q=\frac{\left(x_{2}-a x_{1}\right) x_{3}+(a-1) x_{1} x_{2}}{x_{1}+(a-1) x_{3}-a x_{2}} .
$$

To complete the analysis compute

$$
Q+x_{1}=\frac{\left(x_{2}-x_{1}\right)\left(x_{3}-x_{1}\right)}{x_{1}+(a-1) x_{3}-a x_{2}} .
$$




\section{Likewise}

$$
Q+x_{2}=\frac{a\left(x_{3}-x_{2}\right)\left(x_{2}-x_{1}\right)}{x_{1}+(a-1) x_{3}-a x_{2}} \quad \text { and } \quad Q+x_{3}=\frac{(a-1)\left(x_{3}-x_{2}\right)\left(x_{3}-x_{1}\right)}{x_{1}+(a-1) x_{3}-a x_{2}}
$$

Thus at a point of tangency

$$
\begin{aligned}
y_{1} & =f^{\prime}(Q)=h^{\prime}(Q)=\frac{y_{2}^{a}}{y_{3}^{a-1}} \frac{\left(Q+x_{2}\right)^{a}}{\left(Q+x_{3}\right)^{a-1}} \frac{1}{\left(Q+x_{1}\right)} \\
& =\frac{y_{2}^{a}}{y_{3}^{a-1}} \frac{a^{a}}{(a-1)^{a}} \frac{\left(x_{2}-x_{1}\right)^{a-1}}{\left(x_{3}-x_{1}\right)^{a}}\left(x_{3}-x_{2}\right),
\end{aligned}
$$

which can be rewritten as

$$
y_{1}^{1 / a} y_{3}^{(a-1) / a} \frac{(a-1)^{(a-1) / a}}{a} \frac{x_{3}-x_{1}}{\left(x_{2}-x_{1}\right)^{(a-1) / a}\left(x_{3}-x_{2}\right)^{1 / a}}=y_{2} .
$$

It will now be shown that

$$
\frac{(a-1)^{(a-1) / a}}{a} \frac{x_{3}-x_{1}}{\left(x_{2}-x_{1}\right)^{(a-1) / a}\left(x_{3}-x_{2}\right)^{1 / a}}>1
$$

so that the curve (12) for $y_{2}=y_{2}\left(x_{2}\right)$ lies above the curve

$$
y_{2}=y_{2}\left(x_{2}\right)=y_{1}^{1 / a} y_{3}^{(a-1) / a} .
$$

It suffices to show that

$$
\left(\frac{x_{3}-x_{1}}{a}\right)^{a}>\left(\frac{x_{2}-x_{1}}{a-1}\right)^{a-1}\left(x_{3}-x_{2}\right) .
$$

Thus

$$
\left(\frac{x_{3}-x_{1}}{\ln x_{3}-\ln x_{1}}\left(\ln x_{3}-\ln x_{2}\right)\right)^{\frac{\ln x_{3}-\ln x_{1}}{\ln x_{3}-\ln x_{2}}}>\left(\frac{x_{2}-x_{1}}{\ln x_{2}-\ln x_{1}}\left(\ln x_{3}-\ln x_{2}\right)\right)^{\frac{\ln x_{2}-\ln x_{1}}{\ln x_{3}-\ln x_{2}}}\left(x_{3}-x_{2}\right)
$$

or

$$
\left(\frac{x_{3}-x_{1}}{\ln x_{3}-\ln x_{1}}\right)^{\ln x_{3}-\ln x_{1}}>\left(\frac{x_{3}-x_{2}}{\ln x_{3}-\ln x_{2}}\right)^{\ln x_{3}-\ln x_{2}}\left(\frac{x_{2}-x_{1}}{\ln x_{2}-\ln x_{1}}\right)^{\ln x_{3}-\ln x_{1}} .
$$

Inequality (13) is established in [5].

This establishes Theorem 2 and we see that the whole question of solving (7) for $M, Q$ and $n$ can be summarized in the following diagram (Figure 7): 


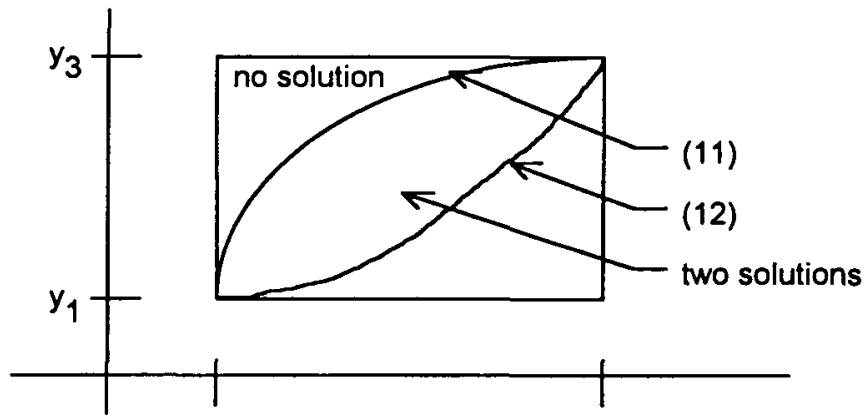

FIGURE 7.

where the curve (11) represents a unique solution.

REMARK 2. Recall from Remark 1 that $Q>0$ above (12), $Q=0$ on (12) and $Q<0$ below (12). To apply these results to the fractal Michaelis-Menten equation it is necessary to have $Q>0$ and so it is of interest to further analyze the curve (12) which forms the boundary to this region.

THEOREM 3. The curve $y_{2}\left(x_{2}\right)=y_{1}^{1 / a} y_{3}^{(a-1) / a}$ is monotone increasing, is concave up if $\frac{y_{3}}{x_{3}}>\frac{y_{1}}{x_{1}}$ and is concave down if $\frac{y_{3}}{x_{3}}<\frac{y_{1}}{x_{1}}$.

This theorem is proven by simple differentiation of

$$
\beta(x)=y_{1}^{\frac{\ln x_{3}-\ln x}{\ln x_{3}-\ln x_{1}}} y_{3}^{\frac{\ln x-\ln x_{1}}{\ln x_{3}-\ln x_{1}}} .
$$

First write

$$
\beta(x)=e^{-\left(\frac{\ln y_{1} \ln x}{\ln x_{3}-\ln x_{1}}\right)} e^{e^{\left(\frac{\ln y_{3} \ln x}{\ln x_{3}-\ln x_{1}}\right)} y_{1}^{\frac{\ln x_{3}}{\ln x_{3}-\ln x_{1}}} y_{3}^{\frac{-\ln x_{1}}{\ln x_{3}-\ln x_{1}}}}
$$

Thus

$$
\beta^{\prime}(x)=\frac{\ln y_{3}-\ln y_{1}}{\ln x_{3}-\ln x_{1}} \frac{1}{x} \beta(x)>0
$$

and

$$
\beta^{\prime \prime}(x)=\left(\frac{\ln y_{3}-\ln y_{1}}{\ln x_{3}-\ln x_{1}}-1\right) \frac{1}{\ln x_{3}-\ln x_{1}} \frac{1}{x^{2}} \beta(x) .
$$

Thus $\beta^{\prime \prime}(x)>0$ if $\frac{y_{3}}{x_{3}}>\frac{y_{1}}{x_{1}}$ and similarly for $\beta^{\prime \prime}(x)<0$. This proves the theorem.

Since it is for $y_{2}>\beta\left(x_{2}\right)$ that positive $M$ and $Q$ can be found, the concavity of $\beta(x)$ determines the "size" of this region. A $\beta(x)$ curve which is concave up provides a larger positive region than the other way around. 


\section{An example}

Finally we use Theorems 1 and 2 to show that sigmoidal data can not always be fit to a fractal Michaelis-Menten curve. We generate sigmoidal data by using the sigmoidal function

$$
w(x)=\frac{5}{1+e^{10-x}}-\frac{5}{1+e^{10}},
$$

which satisfies the conditions $w(0)=0$ and $\lim _{x \rightarrow \infty} w(x)=4.99$ as shown in Figure 8.

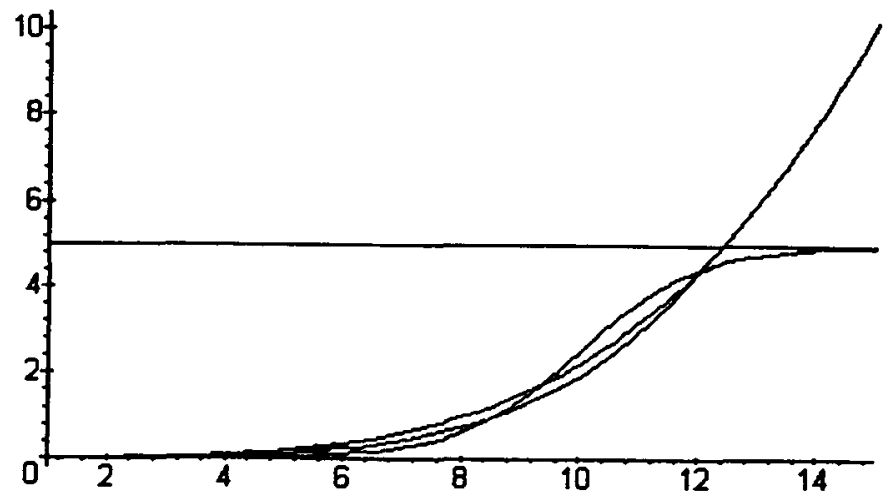

FIGURE 8.

Now adjust the function $y=y_{1}^{1 / a(x)} y_{3}^{1-1 / a(x)}$ so that it passes through the points $(1, w(1))$ and $(12, w(12))$ to obtain the concave (up) curve

$$
y(x)=w(1)^{1-\frac{\ln x}{\ln 12}} w(12)^{\frac{\ln x}{\ln 12}} .
$$

Also define

$$
v(x)=y(x)\left[\frac{(a(x)-1)^{1-1 / a(x)}\left(x_{3}-x_{1}\right)}{a(x)\left(x_{3}-x_{1}\right)^{1-1 / a(x)}\left(x_{3}-x\right)^{1 / a(x)}}\right] .
$$

Both of these curves, $y(x)$ and $v(x)$, are shown in Figure 8 where $w$ is asymptotic to $y=4.99, y$ continues above $y=4.99$ and $v$ is the remaining curve.

On the interval $(1,12)$ only points below $y(x)$ can be uniquely fit to a fractal Michaelis-Menten curve passing through the points $(1, w(1))$ and $(12, w(12))$. Since $w(x)$ lies above $y(x)$ on the interval $(8.65,12)$, then the sigmoidal data $w(x)$ can't be uniquely fit by a fractal Michaelis-Menten curve on the interval $(1,12)$. Theorem 2 indicates the possibility of two solutions for the unknown constants in the fractal 
Michaelis-Menten curve provided that $w(x)<v(x)$ on the interval $(1,12)$. That is not the case here and so no solutions at all exist on the interval $(1,12)$ for the unknown constants in the fractal Michaelis-Menten curve.

\section{Acknowledgement}

The authors wish to thank the referee for many helpful suggestions, especially for pointing out that $Q$ can take on negative values.

\section{References}

[1] K. R. Godfrey, Compartmental models and their applications (Academic Press, N. Y., 1983).

[2] K. R. Godfrey and W. R. Fitch, "The deterministic identifiability of nonlinear pharmacokinetics", $J$. Pharmacokinetics and Biopharmaceutics 12 (1984) 177-191.

[3] J. Heidel and J. Maloney, "When can sigmoidal data be fit to a Hill curve?", J. Austral. Math. Soc., Ser. B, 41 (1999) 83-92.

[4] M. A. Lopez-Quintela and J. Casado, "Revision of the methodology in enzyme kinetics a fractal approach", J. Theor. Biol. 139 (1989) 129-139.

[5] J. Maloney, J. Heidel and J. Pečarić, "A reverse Hölder type inequality for the logarithmic mean and generalizations", J. Austral. Math. Soc., Ser. B, 41 (2000) 401-409.

[6] S. I. Rubinow, Introduction to mathematical biology (John Wiley \& Sons, N. Y., 1975).

[7] J. T. Wong, Kinetics of enzyme mechanisms (Academic Press, N. Y., 1975). 